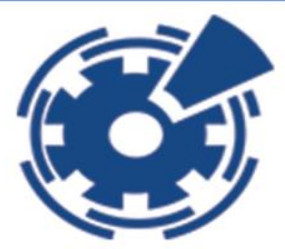

26-28 July, 2019 London, United Kingdom

\title{
In Vitro Study on the Effect on Iron Chelation with Added Natural Iron Chelators to the Diet
}

\author{
Navoda Liyanapathirana ${ }^{1}$, K.K.D.S. Ranaweera ${ }^{2}$, K.D.P.P. Gunathilake ${ }^{3}$ \\ ${ }^{1,2}$ Department of Food Science and Technology, Faculty of Applied Sciences, University of Sri Jayewardenepura, Sri Lanka \\ ${ }^{3}$ Department of Food Science and Technology, Faculty of Livestock, Fisheries and Nutrition, Wayamba University, Sri Lanka
}

\begin{abstract}
Iron overload is rising in the world especially in industrialized countries due to heavy consumption of red meat, alcoholic drinks and iron-fortified foods. Iron chelators remove excess irons in the body and minimize the iron overload related free radical damages. The synthetic chelators used at present are expensive and result in adverse side effects. The study was conducted using in vitro models to assess the viability of dietary interventions on the removal of excess iron in the body through the incorporation of dietary iron chelators to diets as a cheap and feasible alternative treatment for control of iron overload. The fifteen (15) diet plans were formulated based on four ingredients containing natural chelators namely, red rice, fresh milk, turmeric and black tea against a control diet. The designed diets were subjected to in vitro digestion and their effect of iron chelation was assessed through ferrozine assay. The diet plan containing turmeric showed the highest iron chelation ability $(94.47 \pm 0.47 \%)$ closely followed by the diet plan containing turmeric, fresh milk and black tea $(92.58 \pm 0.89 \%)$. Thus, the study concluded that the incorporation of natural iron chelators significant increase the in vitro iron chelation ability $(\mathrm{p}$ value $<0.05$ ) and that turmeric alone or combinations of turmeric, fresh milk and black tea could be used for possible dietary intervention to control iron overload related complications.
\end{abstract}

Keywords: Free radical mediated damages; Iron chelation ability; Iron overload; Natural iron chelators; Stimulated digestion 